\title{
Can MEMS Take Advantage of Advances in Semiconductor Lithography?
}

The title of this editorial is the topic that I selected for my invited talk at the 2010 IEEE MEMS conference. The progress of lithography has helped the semiconductor industry to uphold Moore's law for decades by improving circuit performance while reducing cost. The MEMS industry, having started later than the semiconductor industry and being less developed in economy of scale, can take advantage of the experience of semiconductor patterning to make MEMS patterning cheaper and progress faster. The topic is worth putting some thought into. I accepted the challenge to give the talk and write the proceedings article to share my insight, derived from the development of semiconductor lithography, in the hope of stimulating more innovations to grow MEMS into an industry of equal or greater scale than the semiconductor industry.

In fact, there is a lot of synergy between semiconductors and MEMS. They both use miniaturization for performance and cost reduction. They both have enormous growth potential. Better performance and lower costs enable more applications, which in turn support a larger volume to further promote growth.

Semiconductor lithography has evolved from imaging features at the micrometer scale in the 1970s to the tens of nanometers today, while MEMS started with micrometer features in the 1980s and NEMS is still in a very early research stage. This does not mean NEMS will not become as important as nano-ICs. By taking advantage of our experience in scaling semiconductors, we can reduce the efforts required to scale down MEMS to NEMS.

But there is more to be learned from semiconductor lithography than just shrinking features. For MEMS, lateral patterning is not of primary concern because it is readily available from what has already been developed for the semiconductor industry. On the other hand, the MEMS industry is currently extremely concerned about imaging over large topography. In my presentation, I taught how semiconductor lithographers determine depth of focus (DOF) quantitatively using E-D forest technology. Then, I showed how two-beam imaging can increase the DOF substantially and a new way to take advantage of this setup to produce an oblique image using projection printing instead of proximity printing. The FLEX method to increase DOF by a factor of two to three was included. I also introduced the idea of using two stacked masks to print images at two planes extremely far apart longitudinally.

In the semiconductor industry, a lot of attention is being paid to economy of scale, reduction of cost, elimination of defects, and improvement of yield. These factors are similarly important for the growth of the MEMS industry. Consequently, the current imaging techniques, such as proximity printing, optical projection printing, e-beam direct write, and nanoimprint, will have to converge into optical projection printing for MEMS patterning. With projection printing we can take advantage of the productivity of 8 -in. and 12-in. wafers instead of smaller wafer sizes. Projection printing also has the advantage of low defects and high product yield. To prove the point, steppers and scanners are being used at TSMC to mass-produce micromirror displays, inkjet heads, microfluidic devices, pressure sensors, accelerometers, gyroscopes, microphones, and RF switches. The evolution of projection printing can continue in a better path based on the knowledge we have gained.

Many of the foregoing topics are covered thoroughly and comprehensively in my new book, Optical Lithography: Here Is Why, published by SPIE Press in February this year.

Happy reading!

Burn J. Lin Editor-in-Chief

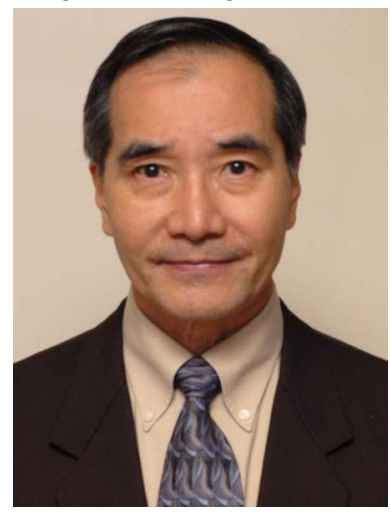

